\title{
Anatomical variations of the abductor pollicis longus: a pilot study
}

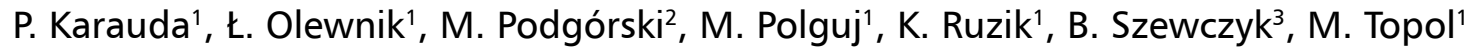 \\ ${ }^{1}$ Department of Normal and Clinical Anatomy, Interfaculty Chair of Anatomy and Histology, \\ Medical University of Lodz, Poland \\ 2Department of Diagnostic Imaging, Polish Mother's Memorial Hospital Research Institute, Lodz, Poland \\ ${ }^{3}$ Department of Clinical Morphology, Medical University of Lodz, Poland
}

[Received: 12 October 2019; Accepted: 3 November 2019]

Background: The abductor pollicis longus (APL) originates from the lateral part of the dorsal surface of the body of the ulna below the insertion of the anconeus muscle, from the interosseous membrane, and from the middle third of the dorsal surface of the body of the radius. However, the number of its accessory bands and their insertion vary considerably.

Materials and methods: Fifty upper limbs (2 paired, 31 male, 19 female) were obtained from adult Caucasian cadavers, and fixed in 10\% formalin solution before examination.

Results: The APL muscle was present in all specimens. The muscles were divided into three main categories, with type II and III being dived into subtypes. Type I was characterised by a single distal attachment, with the tendon inserting to the base of the I metacarpal bone. Type II was characterised by a bifurcated distal attachment, with the main tendon inserting to the base of the first metacarpal bone; this type was divided into three subtypes (a-c). Type III was characterised by the main tendons inserting to the base of the first metacarpal bone, while the accessory band was characterised by mergers (fusion) with other tendons. This type was divided into two subtypes $(a, b)$.

Conclusions: The abductor pollicis longus is characterised by high morphological variability. (Folia Morphol. 2020; 79, 4: 817-822)

Key words: abductor pollicis longus, abductor pollicis longus tendon, tendon graft, anatomical variations

\section{INTRODUCTION}

The forearm bears three muscles that move the thumb, these being the extensor pollicis brevis (EPB) and extensor pollicis longus (EPL), known as the deep extensors, and the abductor pollicis longus muscle (APL). The APL lies immediately below the supinator, and is sometimes united with it. It originates from the lateral part of the dorsal surface of the body of the ulna below the insertion of the anconeus mus- cle, from the interosseous membrane, and from the middle third of the dorsal surface of the body of the radius [10]. The muscle belly of the APL passes obliquely downward and lateralward, becoming a tendon which, together with the tendon of the EPB, runs through a groove on the lateral side of the distal part of the radius and inserts at the radial side of the base of the first metacarpal bone [10]. The APL itself is innervated by the posterior interosseous nerve,

Address for correspondence: Dr. P. Karauda, Department of Normal and Clinical Anatomy, Interfaculty Chair of Anatomy and Histology, Medical University of Lodz, ul. Kościuszki 4, 90-419 Łódź, Poland, e-mail: piotr.karauda@umed.lodz.pl 
a branch of the radial nerve [10]. Its primary function is to abduct the thumb at the carpometacarpal joint, thereby moving the thumb anteriorly, and to assist in extending and rotating the thumb.

The dorsal surface of the hand typically includes a number of muscles divided between six compartments: the EPB and the APL are in the first compartment, the extensor carpi radialis longus and extensor carpi radialis brevis in the second, the EPL in the third, the extensor digitorum and extensor indicis in the fourth, the extensor digiti minimi muscle in the fifth, and the extensor carpi ulnaris in the sixth [10]. Both the first and the third compartment serve as the limits of the "anatomical snuffbox".

The APL demonstrates a high degree of variability with regard to the number of its accessory bands and their insertion $[2-6,9,13-15]$. The presence of multiple tendons originating from the APL is clinically relevant, as it can lead to the development of de Quervain's syndrome, which is caused by stenosing tenosynovitis. In addition, these accessory bands may also serve as a potential source of transplant material, especially for tendon reconstruction [3].

The primary aim of the study is to characterise the morphology of the APL and its accessory bands; however, it is also intended to prepare a classification for distinguishing different types of APL when planning surgical procedures in the area.

\section{MATERIALS AND METHODS}

Fifty upper limbs (31 male, 19 female) were obtained from adult Caucasian cadavers, and fixed in $10 \%$ formalin solution before examination. The upper limbs were stored in a pool of preservative liquid for 3 to 8 years. The cadavers were the property of the Department following donation to the university anatomy program. The inclusion criteria comprised sufficient specimen quality and the lack of evidence of surgical intervention in the examined area; this is needed to allow for complete identification of the tendon insertion. The dissection of the forearm and hand areas was performed according to the standard technique [11, 12]:

Dissection started with the removal of the skin, subcutaneous tissue and superficial fascia. Following this, starting proximal to the retinaculum, the tendon and muscle belly of the APL was exposed and precisely dissected to the bony attachment. Following this, the entire tendon was separated to the distal attachment.

Upon dissection, the following morphological features of the APL were assessed:
- the type of APL tendon insertion (location, shape of tendon insertion);

- morphometric measurements of the APL;

- an electronic digital calliper was used for all measurements (Mitutoyo Corporation, Kawasaki-shi, Kanagawa, Japan). Each measurement was carried out twice with an accuracy of up to $0.1 \mathrm{~mm}$. The size of each band was measured. Consent was obtained from the Local Bioethical Commission to perform the anatomical stage (RNN/09/19/KE).

\section{Ethical approval and consent to participate}

The anatomical protocol of the study was accepted by Bioethics Committee of Medical University of Lodz (resolution RNN/09/19/KE).

The Local Bioethics Commission gave consent for the study (agreement no. RNN/09/19/KE).

The cadavers belong to the Department of Normal and Clinical Anatomy of the Medical University of Lodz.

This article does not contain any studies with human participants or animals performed by any of the authors.

\section{Statistical analysis}

Statistical analysis was performed with Statistica 12 software (StatSoft Polska, Cracow, Poland). The $\chi^{2}$ test with contingency tables was used to evaluate differences between the distribution of APL types according to gender and body side. The normality of the data distribution was checked with the Shapiro-Wilk test. As the data was not normally distributed, the morphological measurements of different APL types were compared using the Mann-Whitney U-test (two groups) or the Kruskal-Wallis test by ranks with a dedicated post hoc test (more than two groups).

A p-value lower than 0.05 was considered significant. The results are presented as mean and standard deviation unless otherwise stated.

\section{RESULTS}

The APL was present in all specimens. Based on its morphology, three main types were distinguished:

- type I: this type possesses a single distal attachment. The tendon inserts to the base of the I metacarpal bone. This type was observed in 5 upper limbs (Fig. 1).

- type II: this type is characterised by a bifurcated distal attachment. The main tendon inserts to the base of the first metatarsal bone. This type was 


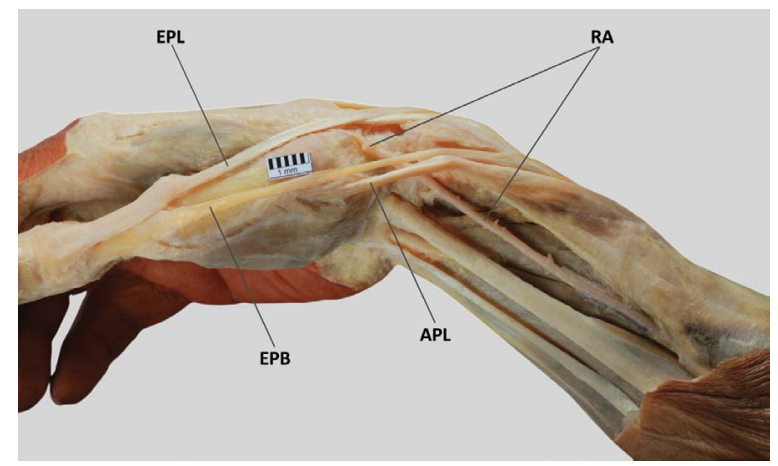

Figure 1. Type I of abductor pollicis longus insertion; APL abductor pollicis longus; EPL — extensor pollicis longus; EPB extensor pollicis brevis; RA — radial artery.

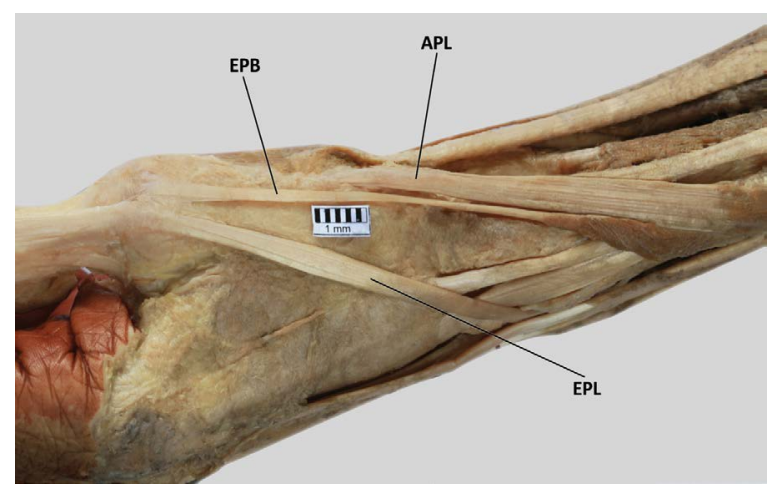

Figure 2. Type lla of abductor pollicis longus insertion; APL abductor pollicis longus; EPL - extensor pollicis longus; EPB extensor pollicis brevis.

present in 6 cases. Two subtypes were determined based on the place of attachment:

- subtype a: the accessory band inserts to the shaft of the first metacarpal bone. This subtype was found in 3 upper limbs (Fig. 2),

- subtype b: the accessory band inserts to the trapezius bone. This subtype occurred in 3 cases (Fig. 3),

- subtype c: the accessory band was divided into two: the first band connected with the abductor pollicis brevis, while the second connected with the opponens pollicis muscle. This subtype was present in 12 cases (Fig. 4);

- type III: this type is also characterised by a double tendon; however, the main band inserts to the base of the first metacarpal bone, while the accessory band fuses with other tendons. It was further divided into two subtypes:

- subtype a: where the accessory band fuses with the APB. This subtype was present in 25 cases (Fig. 5),

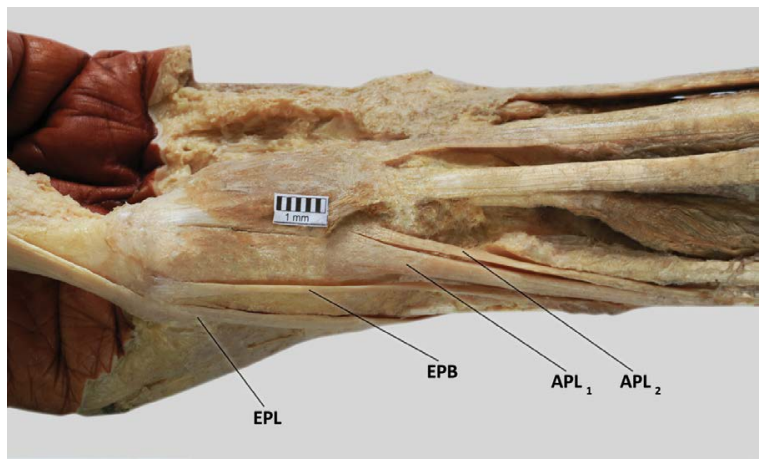

Figure 3. Type llb of abductor pollicis longus insertion; APL abductor pollicis longus; EPL — extensor pollicis longus; EPB — extensor pollicis brevis.

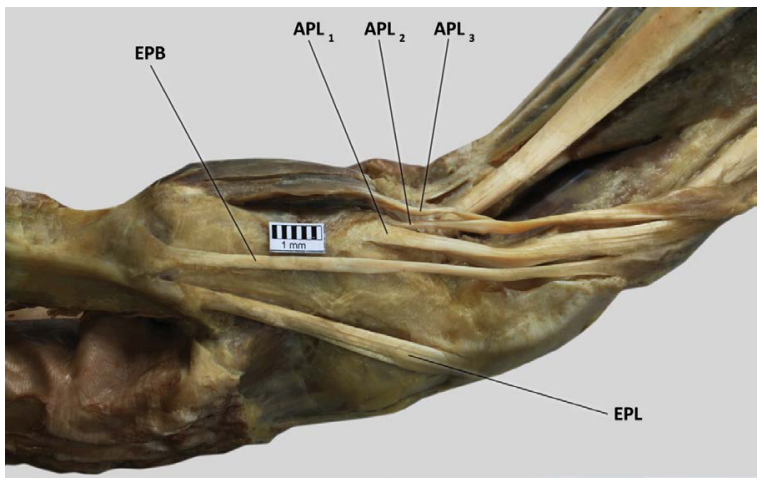

Figure 4. Type Ilc of abductor pollicis longus insertion; APL abductor pollicis longus; EPL — extensor pollicis longus; EPB — extensor pollicis brevis.

- subtype b: where the accessory band fuses with the EPB, and inserts to the trapezius bone. This type was present in 2 cases (Fig. 6).

The morphological characteristics of the tendons and muscle bellies according to body side, gender and each APL type are presented in Tables 1 and 2.

\section{DISCUSSION}

Our work provides a systematic classification of the accessory bands of the APL and their type of insertion. The proposed classification is the first to systematize the relevant APL insertion variants into three main types (I-III), with type II and III being divided into two or three subtypes.

Different types of accessory band insertions have been described in the literature [1-6, 9, 13-15], and such great morphological variability is believed to have an embryological basis. The APL and EPB differentiate from a common muscle mass and are characterised by different anatomies in other primates [7], with a continuum of differentiation of 


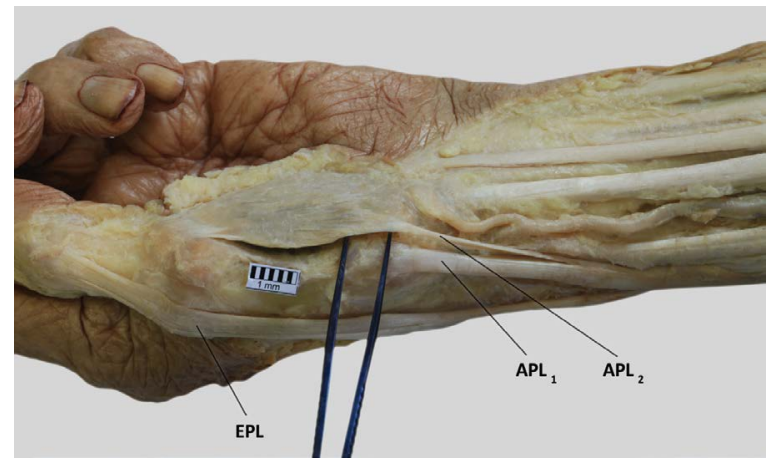

Figure 5. Type Illa of abductor pollicis longus insertion; APL abductor pollicis longus; EPL — extensor pollicis longus.

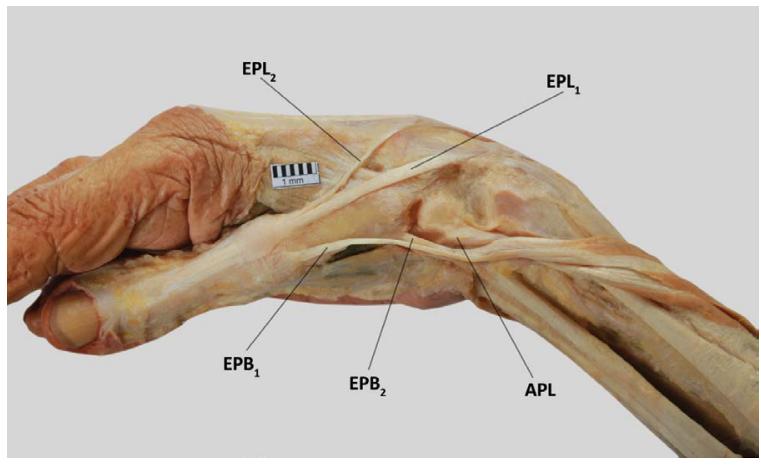

Figure 6. Type Illb of abductor pollicis longus insertion; APL abductor pollicis longus; EPL — extensor pollicis longus; EPB extensor pollicis brevis.

Table 1. The morphological characteristics of the abductor pollicis longus tendons and muscle bellies according to body side and gender

\begin{tabular}{|c|c|c|c|c|c|c|c|c|c|c|}
\hline \multirow[t]{3}{*}{ Parameter } & \multicolumn{4}{|c|}{ Gender } & \multirow[t]{3}{*}{$\mathbf{P}$} & \multicolumn{4}{|c|}{ Body side } & \multirow[t]{3}{*}{$\mathbf{P}$} \\
\hline & \multicolumn{2}{|c|}{ Female } & \multicolumn{2}{|c|}{ Male } & & \multicolumn{2}{|c|}{ Right } & \multicolumn{2}{|c|}{ Left } & \\
\hline & Mean & SD & Mean & SD & & Mean & SD & Mean & SD & \\
\hline Forearm length & 25.19 & 1.61 & 27.03 & 1.35 & 0.0005 & 26.07 & 1.61 & 26.71 & 1.76 & 0.1948 \\
\hline $\begin{array}{l}\text { Width of the main tendon in the } \\
\text { musculo-tendinous junction }\end{array}$ & 5.11 & 1.66 & 5.83 & 1.67 & 0.1418 & 5.40 & 1.73 & 5.75 & 1.64 & 0.3532 \\
\hline $\begin{array}{l}\text { Width of the accessory tendon in the } \\
\text { musculo-tendinous junction }\end{array}$ & 3.40 & 0.87 & 3.55 & 1.33 & 0.8836 & 3.07 & 0.98 & 3.86 & 1.18 & 0.1939 \\
\hline $\begin{array}{l}\text { Thickness of the main tendon in the } \\
\text { musculo-tendinous junction }\end{array}$ & 1.55 & 0.41 & 1.91 & 0.52 & 0.0258 & 1.74 & 0.39 & 1.83 & 0.62 & 0.4756 \\
\hline $\begin{array}{l}\text { Thickness of the accessory tendon } \\
\text { in the musculo-tendinous junction }\end{array}$ & 1.49 & 0.52 & 1.41 & 0.21 & 0.6604 & 1.39 & 0.36 & 1.49 & 0.38 & 0.6304 \\
\hline Length of the main tendon & 57.37 & 19.04 & 68.15 & 12.71 & 0.0576 & 63.44 & 19.59 & 64.84 & 10.60 & 0.9299 \\
\hline Length of the tendon $2 \mathrm{~A}$ & 30.91 & 21.27 & 50.66 & 20.16 & 0.0406 & 43.05 & 22.78 & 42.80 & 23.09 & 0.9755 \\
\hline Length of the tendon $2 \mathrm{~B}$ & 20.10 & 14.70 & 33.15 & 24.70 & 0.2225 & 28.57 & 25.34 & 27.07 & 19.31 & 0.7363 \\
\hline Width of the main tendon distal attachment & 4.61 & 1.91 & 4.09 & 1.49 & 0.4898 & 4.23 & 1.81 & 4.36 & 1.51 & 0.6655 \\
\hline Width of the tendon $2 \mathrm{~A}$ distal attachment & 3.50 & 1.45 & 4.40 & 2.71 & 0.8397 & 4.38 & 2.80 & 3.72 & 1.71 & 0.6784 \\
\hline Width of the tendon $2 \mathrm{~B}$ distal attachment & 2.99 & 1.23 & 3.36 & 1.36 & 0.7871 & 3.26 & 1.13 & 3.15 & 1.53 & 0.7040 \\
\hline Thickness of the main tendon at the distal attachment & 1.22 & 0.32 & 1.41 & 0.56 & 0.2416 & 1.26 & 0.44 & 1.43 & 0.53 & 0.2748 \\
\hline Thickness of the tendon $2 \mathrm{~A}$ at the distal attachment & 1.41 & 0.86 & 1.57 & 0.80 & 0.5144 & 1.46 & 0.87 & 1.56 & 0.77 & 0.6312 \\
\hline Thickness of the tendon 2B at the distal attachment & 1.22 & 0.61 & 1.36 & 0.59 & 0.5369 & 1.33 & 0.72 & 1.28 & 0.42 & 1.0000 \\
\hline Length of the accessory tendon & 34.67 & 25.58 & 57.01 & 22.64 & 0.0491 & 51.85 & 24.02 & 46.18 & 28.86 & 0.6836 \\
\hline Width of the accessory tendon origin & 2.47 & 0.96 & 2.99 & 1.15 & 0.3364 & 2.95 & 1.10 & 2.60 & 1.12 & 0.5150 \\
\hline Thickness of the accessory tendon origin & 1.07 & 0.44 & 1.26 & 0.48 & 0.3983 & 1.24 & 0.48 & 1.11 & 0.46 & 0.6304 \\
\hline Width of the accessory tendon at the distal attachment & 3.53 & 1.60 & 3.18 & 0.95 & 0.7486 & 3.28 & 1.30 & 3.32 & 0.97 & 0.6919 \\
\hline $\begin{array}{l}\text { Thickness of the accessory tendon at the distal } \\
\text { attachment }\end{array}$ & 0.99 & 0.44 & 1.15 & 0.34 & 0.1999 & 1.08 & 0.33 & 1.13 & 0.45 & 0.8874 \\
\hline
\end{tabular}

SD - standard deviation

common muscle mass observed between species: the APL inserts to the radial side of the shaft of the first metacarpal bone in chimpanzees, but inserts to the trapezium bone in gorillas. Phylogenetically, the process is still visible in infancy and therefore it is not surprising that anatomical variations are found in man [7].

Anatomical variations of APL tendons were first described in detail by Anson [1], who reported seven different types, the most common being one char- 
Table 2. The morphological characteristics of the tendons and muscle bellies of each abductor pollicis longus (APL) type

\begin{tabular}{|c|c|c|c|c|c|c|c|}
\hline \multirow[t]{3}{*}{ Parameter } & \multicolumn{6}{|c|}{ APL type } & \multirow[t]{3}{*}{$\mathbf{P}$} \\
\hline & \multicolumn{2}{|c|}{ I } & \multicolumn{2}{|c|}{ II } & \multicolumn{2}{|c|}{ III } & \\
\hline & Mean & SD & Mean & SD & Mean & SD & \\
\hline Forearm length & 25.60 & 1.82 & 26.47 & 1.61 & 26.43 & 1.75 & 0.5863 \\
\hline Width of the main tendon in the musculo-tendinous junction & 6.81 & 1.88 & 4.79 & 1.34 & 5.83 & 1.69 & 0.0325 \\
\hline Width of the accessory tendon in the musculo-tendinous junction & & & 3.49 & 1.13 & & & - \\
\hline Thickness of the main tendon in the musculo-tendinous junction & 1.92 & 0.37 & 1.68 & 0.54 & 1.82 & 0.51 & 0.3167 \\
\hline Thickness of the accessory tendon in the musculo-tendinous junction & & & 1.44 & 0.36 & & & - \\
\hline Length of the main tendon & 65.03 & 10.20 & 69.73 & 13.09 & 60.10 & 17.98 & 0.0972 \\
\hline Length of the tendon $2 \mathrm{~A}$ & & & 42.60 & 25.03 & 43.68 & 16.52 & 0.9202 \\
\hline Length of the tendon $2 \mathrm{~B}$ & & & 33.04 & 24.41 & 18.12 & 11.54 & 0.2090 \\
\hline Width of the main tendon at the distal attachment & 5.12 & 1.45 & 4.15 & 1.76 & 4.21 & 1.64 & 0.2261 \\
\hline Width of the tendon $2 \mathrm{~A}$ at the distal attachment & & & 2.99 & 1.12 & 5.56 & 2.78 & 0.0037 \\
\hline Width of the tendon $2 \mathrm{~B}$ at the distal attachment & & & 2.47 & 0.86 & 4.33 & 0.98 & 0.0014 \\
\hline Thickness of the main tendon at the distal attachment & 1.52 & 0.36 & 1.16 & 0.45 & 1.44 & 0.51 & 0.1666 \\
\hline Thickness of the tendon $2 \mathrm{~A}$ at the distal attachment & & & 1.25 & 0.72 & 1.88 & 0.82 & 0.0189 \\
\hline Thickness of the tendon $2 \mathrm{~B}$ at the distal attachment & & & 0.96 & 0.36 & 1.83 & 0.46 & 0.0014 \\
\hline Length of the accessory tendon & & & 40.63 & 31.52 & 50.93 & 25.20 & 0.5524 \\
\hline Width of the accessory tendon origin & & & & & 2.82 & 1.10 & - \\
\hline Thickness of the accessory tendon origin & & & & & 1.20 & 0.46 & - \\
\hline Width of the accessory tendon at the distal attachment & & & & & 3.29 & 1.17 & - \\
\hline Thickness of the accessory tendon at the distal attachment & & & & & 1.10 & 0.37 & - \\
\hline
\end{tabular}

SD - standard deviation

acterised by insertion into both the first metacarpal and the greater multangular bones (45.8\%) [1]. In the second most common type, the APL inserts into the first metacarpal and, additionally to the abductor pollicis brevis and the greater multangular (14.1\%) [1]. The third most frequent insertion was into the first metacarpal bone and the APB (12.8\%). The other four types were characterised by a variable distal attachment, such as fusions with the opponens pollicis and insertion to the styloid process of the radii and the volar carpal ligament; however, these variations were much more rarely observed and were not found among our specimens [1]. Differences between variations described by other authors and these found in our work are presented in Table 3.

Our prosed classification is intended to assist clinicians while preparing for surgery or during diagnosis. In this case, a classification based on ten, eight or even seven types is too unwieldy; a simpler and more practical approach would be to divide the types based on single, bifurcated (with subtypes) and trifurcated types (with subtypes), as in the proposed classification.
A good knowledge of the variations of the APL is important from the clinical point of view. For example, de Quervain's tenosynovitis often develops due to repetitive and continued strain of the APL and EPB tendons as they pass under a thickened and swollen extensor retinaculum [8]. Symptoms include pain at the radial side of the wrist, spasms, tenderness, an occasional burning sensation in the hand and swelling over the thumb side of the wrist, and difficulty gripping with the affected side of the hand. The onset is often gradual and pain is made worse by movement of the thumb and wrist, and may radiate to the thumb or the forearm [8]. The presence of additional APL bands running in separate sheaths may influence the choice of treatment, as they can reduce the penetration of steroids used in conservative approaches and prevent full surgical release, thus resulting in failure. Similarly, abnormal insertion into the phalanx or metacarpal can cause trapezio-metacarpal subluxation or arthritis. In addition, $A B L$ is also commonly used as material for tendon grafts; hence, the presence of additional bands might affect the grafting procedure, the quality of the graft and the remaining function of the thumb $[3,8]$. 
Table 3. The differences between the classifications given in the present study and those of other authors

\begin{tabular}{|c|c|c|c|c|c|c|c|c|c|c|}
\hline $\begin{array}{l}\text { Types of insertion } \\
\text { of the APL }\end{array}$ & $\begin{array}{c}\text { Tewari } \\
\text { et al. [\%] }\end{array}$ & $\begin{array}{l}\text { Mansur } \\
\text { et al. [\%] }\end{array}$ & $\begin{array}{c}\text { Rosa } \\
\text { et al. [\%] }\end{array}$ & $\begin{array}{l}\text { Kocabiyik } \\
\text { et al. [\%] }\end{array}$ & $\begin{array}{l}\text { Gurude } \\
\text { et al. [\%] }\end{array}$ & $\begin{array}{l}\text { Sarikcioglu } \\
\text { et al. [\%] }\end{array}$ & $\begin{array}{c}\text { Rabi } \\
\text { et al. [\%] }\end{array}$ & $\begin{array}{l}\text { Sarikcioglu } \\
\text { et al. [\%] }\end{array}$ & $\begin{array}{c}\text { Mehta } \\
\text { et al. [\%] }\end{array}$ & $\begin{array}{c}\text { Present } \\
{[\%]}\end{array}$ \\
\hline Type I & 4 & & & & & & & & & 10 \\
\hline Type lla & 4 & & & & & & & & & 6 \\
\hline Type llb & 58 & & & & Case & & & & & 6 \\
\hline Type Ilc & & & & & & & & & & 26 \\
\hline Type Illa & & & & Case & & & & & & 48 \\
\hline Type IIlb & & & & & & & & & & 4 \\
\hline
\end{tabular}

APL — abductor pollicis longus

\section{Limitations of the study}

The present study has some limitations: no sample size calculation was performed and a relatively low number of dissected limbs were examined. Nevertheless, based on available material, we were able to propose classification that can cover all variations of the APL and can serve as a basis for further studies, and as a foundation for communication with surgeons in clinical practice. One final limitation is that the study explores only variations of the APL and not the EPB, despite the fact that it might also present variants with a significant influence important on the aetiology and treatment of de Quervain's tenosynovitis.

\section{CONCLUSIONS}

The APL is characterised by high morphological variability associated with the presence of additional bands and fusion with neighbouring muscles. Our proposed classification is simple and serves as a foundation for further development with rare variants.

\section{Acknowledgements}

The authors wish to express their gratitude to all those who donated their bodies to medical science.

\section{REFERENCES}

1. Anson B. An Atlas of Human Anatomy. WB Saunders Company, Philadelphia 1963.

2. Bharambe V, Patel D, Rao Manvikar P, et al. A Study of Extensor Pollicis longus and brevis and Abductor pollicis longus from the perspective of Evolution. J Med Res. 2017; 3(3): 146-150, doi: 10.31254/jmr.2017.3311.

3. Bravo $E$, Barco R, Bullón A. Anatomic study of the abductor pollicis longus: a source for grafting material of the hand. Clin Orthop Relat Res. 2010; 468(5): 1305-1309, doi: 10.1007/ s11999-009-1059-4, indexed in Pubmed: 19760470.

4. Coleman SS, McAfee DK, Anson BJ. The insertion of the abductor pollicis longus muscle; an anatomical study of 175 specimens. Q Bull Northwest Univ Med Sch. 1953; 27(2): 117-122, indexed in Pubmed: 13056139.
5. El-Beshbishy RA, Abdel-Hamid GA. Variations of the abductor pollicis longus tendon: an anatomic study. Folia Morphol. 2013; 72(2): 161-166, doi: 10.5603/ fm.2013.0027, indexed in Pubmed: 23740505.

6. Fabrizio PA, Clemente FR. A variation in the organization of abductor pollicis longus. Clin Anat. 1996; 9(6): 371-375, doi: 10.1002/(SICI)1098-2353(1996)9:6<371::AIDCA2>3.0.CO;2-E, indexed in Pubmed: 8915615.

7. Giles KW. Anatomical variations affecting the surgery of de Quervain's disease. J Bone Joint Surg Br. 1960; 42-B: 352-355, indexed in Pubmed: 13850049.

8. Goel R, Abzug JM. de Quervain's tenosynovitis: a review of the rehabilitative options. Hand. 2015; 10(1): 1-5, doi: 10.1007/ s11552-014-9649-3, indexed in Pubmed: 25762881.

9. Mahakkanukrauh $P$, Mahakkanukrauh $C$. Incidence of a septum in the first dorsal compartment and its effects on therapy of de Quervain's disease. Clin Anat. 2000; 13(3): 195-198, doi: 10.1002/(SICI)10982353(2000)13:3<195::AID-CA6>3.0.CO;2-V, indexed in Pubmed: 10797626.

10. Moore K, Arthur F, Dalley I. Clinically Oriented Anatomy. Lippincott Williams \& Wilkins 2013.

11. Olewnik $\measuredangle$, Podgórski $M$, Polguj $M$, et al. Anatomical variations of the pronator teres muscle in a Central European population and its clinical significance. Anat Sci Int. 2018; 93(2): 299-306, doi: 10.1007/s12565-017-0413-y, indexed in Pubmed: 28849397.

12. Olewnik $Ł$, Wysiadecki G, Polguj M, et al. Anatomical variations of the palmaris longus muscle including its relation to the median nerve: a proposal for a new classification. BMC Musculoskelet Disord. 2017; 18(1): 539, doi: 10.1186/ s12891-017-1901-x, indexed in Pubmed: 29258498.

13. Opreanu RC, Wechter J, Tabbaa H, et al. Anatomic variations of the first extensor compartment and abductor pollicis longus tendon in trapeziometacarpal arthritis. Hand. 2010; 5(2): 184-189, doi: 10.1007/s11552-0099234-3, indexed in Pubmed: 19834771.

14. Thwin SS, Fazlin F, Than M. Multiple variations of the tendons of the anatomical snuffbox. Singapore Med J. 2014; 55(1): 37-40, doi: 10.11622/smedj.2013216, indexed in Pubmed: 24452976.

15. Tripathy SK, Tewari J, Mishra PR, et al. Anatomical variation of abductor pollicis longus in Indian population: A cadaveric study. Indian J Orthop. 2015; 49(5): 549-553, doi: 10.4103/0019-5413.164038, indexed in Pubmed: 26538762. 